\title{
Probability of primordial black hole formation and its dependence on the radial profile of initial configurations
}

\author{
J. C. Hidalgo ${ }^{\dagger}$ and A. G. Polnarev ${ }^{\ddagger *}$ \\ Astronomy Unit, School of Mathematical Sciences, \\ Queen Mary College, \\ University of London, Mile End Road, \\ London E1 4NS, United Kingdom
}

(Dated: November 6, 2018)

\begin{abstract}
In this paper we derive the probability of the radial profiles of spherically symmetric inhomogeneities in order to provide an improved estimation of the number density of primordial black holes (PBHs). We demonstrate that the probability of $\mathrm{PBH}$ formation depends sensitively on the radial profile of the initial configuration. We do this by characterising this profile with two parameters chosen heuristically: the amplitude of the inhomogeneity and the second radial derivative, both evaluated at the centre of the configuration. We calculate the joint probability of initial cosmological inhomogeneities as a function of these two parameters and then find a correspondence between these parameters and those used in numerical computations of PBH formation. Finally, we extend our heuristic study to evaluate the probability of $\mathrm{PBH}$ formation taking into account for the first time the radial profile of curvature inhomogeneities.
\end{abstract}

\section{INTRODUCTION}

The idea that large amplitude matter overdensities in the universe could have collapsed through selfgravity to form primordial black holes (PBHs) was first put forward by Zeldovich and Novikov [1], and then independently by Hawking [2], more than three decades ago. This theory suggests that large amplitude inhomogeneities in the very early universe overcome internal pressure forces and collapse to form black holes. A lower threshold for the amplitude of such inhomogeneities $\delta_{\mathrm{th}} \equiv(\delta \rho / \rho)_{\mathrm{th}}$, was first provided by Carr [3], giving $\delta_{\mathrm{th}} \approx 1 / 3$ at the time of radiation domination. This value was found by comparing the Jeans length of the overdensity with the scale of the cosmological horizon at the time of formation.

The mass fraction of the universe turning into $\mathrm{PBHs}$ of mass $M$ at their formation time, $\beta_{\mathrm{PBH}}(M)$, is computed using the probability density function (PDF) of the relevant field of inhomogeneities, which is provided by the cosmological theory. The mass fraction $\beta_{\mathrm{PBH}}(M)$ is customarily given by the integral of this PDF over the amplitude $\delta \equiv \delta \rho / \rho$, with a lower limit equal to $\delta_{\text {th }}[3,4]$.

The probability of $\mathrm{PBH}$ formation is a useful tool to constrain the mean amplitude of inhomogeneities on scales which cannot be probed by any other methods. The PBH contribution to the energy density increases with time during the radiation-dominated epoch. For this reason, the PBHs formed considerably before the end of radiation domination are the most relevant to cosmology [5-10]. We will focus our study on these kind of PBHs and assume that the background matter at the time of PBH formation is radiationdominated. To make this cosmological tool more precise, we must improve the calculations of the probability of PBH formation. This demands a more accurate evaluation of the threshold value $\delta_{\text {th }}$, or the equivalent curvature inhomogeneity $\mathcal{R}_{\text {th }}$ [11-16]. In search of these values, it was evident that the process of PBH formation depends on the pressure gradients in the collapsing configuration in addition to the amplitude [16-18]. It was also found that such pressure gradients can modify the value of $\delta_{\text {th }}$ significantly. Hence, when calculating the probability of $\mathrm{PBH}$ formation, one should consider the shape and radial profile of the initial configurations. These profiles are directly related with the internal pressure gradients. This is the main motivation for the present work. Previous studies, concerning the probability of PBH formation, take the amplitude of perturbations to be the only parameter determining the probability density. In addition to that, we include for the first time a parameter related to the slope of curvature profile at the edge of the configuration ${ }^{1}$.

\footnotetext{
*†c.hidalgo@qmul.ac.uk; ła.g.polnarev@qmul.ac.uk

${ }^{1}$ In the context of dark matter haloes the question of initial profiles is effectively irrelevant because galaxies are formed from pressureless configurations. The density profiles and shapes of virialized haloes result from the evolution of the initial high peaks and are not linked to the profile of initial configurations that we investigate here (see e.g. [19] for a review on the
} 
In this paper, we calculate the probability of finding a curvature configuration with a given radial profile. As follows from [21-25], PBH formation takes place only from nearly spherical configurations, so in this paper we restrict ourselves to the spherically symmetric case. In this first approximation we describe the radial profiles by introducing two parameters: the central amplitude of the curvature inhomogeneity $\mathcal{R}(\mathbf{r}=0)$ and the second radial derivative at the centre $\mathcal{R}^{\prime \prime}(\mathbf{r}=0)$, which is chosen mainly to avoid technical difficulties. The introduction of these parameters is a first step towards the full parametrisation of profiles in terms of even derivatives at the centre of configurations, i.e. in terms of $\mathcal{R}^{(2 n)}(0)$ (the odd derivatives $\mathcal{R}^{(2 n+1)}(0)$ are all zero due to the assumed spherical symmetry). In the future, with the results from more accurate codes simulating the formation of PBHs, we will have at hand a larger number of conditions for the collapse of a curvature profile. An equal number of parameters will be required for the complete description of these profiles and the probability of finding them. In the meantime, only families of curvature profiles described by two parameters are available. We consequently limit ourselves to the two-parametric description of initial curvature profiles.

The central amplitude $\mathcal{R}(0)$ has been used in previous calculations of gravitational collapse [16], and the probability of $\mathrm{PBH}$ formation $[8,26]$. In the present paper we compute the probability to find a given configuration as a function of the two parameters $\left[\mathcal{R}(0), \mathcal{R}^{\prime \prime}(0)\right]$. We subsequently illustrate how this twoparametric probability is used to correct the probability of $\mathrm{PBH}$ formation. Such an exercise is presented for illustration purposes. The results, based on a non-rigorous but physically meaningful determination of the parameters which describe the initial profiles, show how the corrections to $\beta_{\mathrm{PBH}}$ are significant and they will be considered in more detail in future studies of PBH formation.

This paper is organised as follows. In Section II we calculate the joint probability distribution for $\mathcal{R}(0)$ and $\mathcal{R}^{\prime \prime}(0)$. In Section III, we relate these parameters to those used in the most recent numerical computations of $\mathrm{PBH}$ formation. In Section IV we present the total probability of PBH formation, integrating the probability distribution derived in Section II over the relevant region of parameter space $\left[\mathcal{R}(0), \mathcal{R}^{\prime \prime}(0)\right]$. In Section V we summarise our results and discuss future research in this area.

\section{PROBABILITY OF RADIAL PROFILE PARAMETERS OF COSMOLOGICAL PERTURBATIONS}

The most striking prediction of the theory of cosmological inflation is that initial quantum fluctuations are transfered into the inhomogeneities and structures observed in the universe today. After inflation, the universe is mostly flat with inhomogeneities of small amplitude on average (for a review, see [27]). Some of the inhomogeneous regions, present high amplitude (non-linear inhomogeneities) and these are the object of study in the present paper. Formally, the high amplitude inhomogeneous profiles describing configurations which collapse into PBHs are not perturbations. However, such regions are included in the statistics of random primordial curvature perturbations. That is, the statistics of random fields can be used to estimate the probability of finding high amplitude inhomogeneities.

To describe large-amplitude inhomogeneities, we consider the non-linear curvature field $\mathcal{R}(t, r)$, as first described in [28]. The non-linear curvature $\mathcal{R}(r, t)$ represents the relative expansion of a given local patch of the universe with respect to its neighbouring patches [29]. It is described by the metric

$$
\mathrm{d} s^{2}=-N^{2}(t, \mathbf{r}) \mathrm{d} t^{2}+a^{2}(t) \mathrm{e}^{2 \mathcal{R}(t, \mathbf{r})} \tilde{\gamma}_{i j}\left(\mathrm{~d} r^{i}+N^{i}(t, \mathbf{r}) \mathrm{d} t\right)\left(\mathrm{d} r^{j}+N^{j}(t, \mathbf{r}) \mathrm{d} t\right),
$$

where $a(t)$ is the scale factor and the gauge dependent functions $N$ and $N^{i}$ are the lapse function and shift vector, respectively. These variables are determined by algebraic constraint equations in terms of the matter density $\rho$ and pressure $p$ and the metric variables $\mathcal{R}, a$ and $\tilde{\gamma}_{i j}$.

In this work we consider the non-linear configurations which correspond to non-zero and large $\mathcal{R}$ inside some restricted volume, and zero outside, where the expansion of the universe follows the background Friedmann-Robertson-Walker (FRW) solution. There are several advantages of working with metric (1). First, $\mathcal{R}$ is defined as a gauge-invariant combination of metric and matter variables [30]. Second, with the aid of the gradient expansion of the metric quantities [28, 31-33], $\mathcal{R}(\mathbf{r}, t)$ is presented in the Einstein equations in a non-perturbative way. Finally, $\mathcal{R}$ does not depend on time for scales larger than the cosmological horizon, as proved in $[29,34]$ and in a more general case in [35]. In the present paper we work in the superhorizon régime, where the field $\mathcal{R}(\mathbf{r})$ can be assumed to be time-independent. 
The primordial field of random perturbations we use follows a Gaussian probability distribution. In other words, we assume that the probability of finding a curvature perturbation $\mathcal{R}$ of mean amplitude $\vartheta$ is given by the probability density function (PDF)

$$
\mathbb{P}[\vartheta] \propto \exp \left(-\frac{\vartheta^{2}}{2 \Sigma_{\mathcal{R}}^{2}}\right),
$$

where $\Sigma_{\mathcal{R}}$ is the dispersion of the perturbation field $\mathcal{R}$, defined in terms of the two-point correlation function by

$$
\Sigma_{\mathcal{R}}^{2}\left(r_{H}\right)=\langle\mathcal{R}(r) \mathcal{R}(r)\rangle=\int \mathrm{d} \ln k \mathcal{W}^{2}\left(k, k_{H}\right) \mathcal{P}(k),
$$

where $\mathcal{W}\left(k, k_{H}\right)$ is the window function which smooths the field over spherical regions of size $r_{H}=2 \pi / k_{H}$, the Hubble radius. The power spectrum of $\mathcal{R}, \mathcal{P}(k)$, is an output of the underlying cosmological model, as reviewed in [27].

In more general cases, PDFs include the contribution of higher-order correlations (i.e. $\langle\mathcal{R} \mathcal{R} \mathcal{R}\rangle$ and all other correlations). To calculate such PDFs, a new formalism is required, such as that developed in [36]. Several studies have shown that the non-Gaussian correlations can sensibly modify the PDF of the amplitude of perturbations and consequently the number count of astrophysical objects [37-39] and PBHs [40-43] when large non-Gaussianities arise in the primordial field of curvature fluctuations [44-47]. Here however, we restrict ourselves to the Gaussian case where the PDF presents the form of Eq. (2).

In the following we calculate the joint probability of finding an amplitude $\mathcal{R}(0)$ and the second derivative

$$
\mathcal{R}^{\prime \prime}(0)=\left[\frac{\partial^{2}}{\partial r^{2}} \mathcal{R}(r)\right]_{r=0},
$$

at the centre, using the method developed in [36]. In order to compute the probability of a specific property of $\mathcal{R}(\mathbf{r})$, we integrate the original PDF, which encodes all the information about the field, with the Dirac $\delta$-functions of relevant arguments. In particular, the probability that $\mathcal{R}(0)=\vartheta_{0}$ is given by,

$$
\mathbb{P}\left(\vartheta_{0}\right)=\int[d \mathcal{R}] \mathbb{P}(\mathcal{R}) \delta\left[\mathcal{R}(0)-\vartheta_{0}\right],
$$

were $[d \mathcal{R}]$ indicates integration over all possible configurations $\mathcal{R}(\mathbf{k})$ in Fourier space. Hereafter we consider $\mathcal{R}(0)$ and $\mathcal{R}^{\prime \prime}(0)$ as statistically independent parameters. Hence, the probability of having $\mathcal{R}^{\prime \prime}(0)=\vartheta_{2}$, is given by the integral

$$
\mathbb{P}\left(\vartheta_{2}\right)=\int[d \mathcal{R}] \mathbb{P}(\mathcal{R}) \delta\left[\mathcal{R}^{\prime \prime}(0)-\vartheta_{2}\right]
$$

In the rest of this section we show roughly how this method works. The details of the following results are presented in appendices A and B. First we expand the smoothed curvature perturbation profile $\mathcal{R}(\mathbf{r})$ in terms of spherical harmonic functions:

$$
\mathcal{R}(\mathbf{r})=\int \frac{d^{3} k}{(2 \pi)^{3}} \mathcal{R}(\mathbf{k}) \exp (\mathrm{ik} \cdot \mathbf{r}),
$$

with

$$
\mathcal{R}(\mathbf{k})=\sum_{\ell=0}^{\infty} \sum_{m=-\ell}^{\ell} \sum_{n=1}^{\infty} \mathcal{R}_{\ell \mid n}^{m} Y_{\ell m}(\theta, \phi) \psi_{n}(k) .
$$

Here $Y_{\ell m}$ are the usual spherical harmonics on the unit 2-sphere and $\psi_{n}(k)$ are a complete and orthogonal set of functions in an arbitrary finite interval $0<k<\Lambda$ (for an explicit expression of $\psi(k$ ) see appendix A). It is worth mentioning that the cutoff $\Lambda$ is imposed to artificially compactify the momentum space. This allows us to provide an explicit definition of the functions $\psi_{n}(k)$ and a complete set of functions $\psi$ for the expansion of $\mathcal{R}(\mathbf{k})$. In turn this condition allows a regularisation of the path integral $\int[d \mathcal{R}]$ by considering the harmonic expansion (8) in a finite interval in Fourier-space $0<k<\Lambda$. At the end of the calculation we can take the limit $\Lambda \rightarrow \infty$ and the results will remain unchanged. The coefficients in the expansion are 
generically complex, so we separate real and imaginary part introducing $\mathcal{R}_{\ell \mid n}^{m}=a_{\ell \mid n}^{m}+\mathrm{i} b_{\ell \mid n}^{m}$. The reality condition for the curvature field, $\overline{\mathcal{R}}(\mathbf{k})=\mathcal{R}(-\mathbf{k})$, is met when

$$
\begin{gathered}
a_{\ell \mid n}^{-m}=(-1)^{\ell+m} a_{\ell \mid n}^{m}, \\
b_{\ell \mid n}^{-m}=(-1)^{\ell+m+1} b_{\ell \mid n}^{m} .
\end{gathered}
$$

In particular, the $m=0$ modes require $a_{\ell \mid n}^{0}$ and $b_{\ell \mid n}^{0}$ to be zero for odd and even $\ell$, respectively. After evaluating the expansion (7)-(8) at $\mathcal{R}(\mathbf{r}=0)$, we can use the relation,

$$
\int d \Omega Y_{\ell}^{m}(\theta, \phi)=\sqrt{4 \pi} \delta^{m 0} \delta_{\ell 0}
$$

where $d \Omega=\sin (\theta) d \theta d \phi$, and integrate Eq. (7) to obtain

$$
\begin{aligned}
\mathcal{R}(0)=\left.\int \frac{d^{3} k}{(2 \pi)^{3}} \mathcal{R}(k) \exp (\mathrm{ik} \cdot \mathbf{r})\right|_{r=0} & =\int \frac{k^{2} d k}{(2 \pi)^{3}} \sum_{\ell=0}^{\infty} \sum_{m=0}^{\ell} \sum_{n=1}^{\infty} \mathcal{R}_{\ell \mid n}^{m}\left(\sqrt{4 \pi} \delta_{\ell 0} \delta_{m 0}\right) \psi_{n}(k), \\
& =\sum_{n=1}^{\infty} a_{0 \mid n}^{0} \int \frac{d k}{\sqrt{\pi}(2 \pi)^{2}} \psi_{n}(k) k^{2}=\vartheta_{0} .
\end{aligned}
$$

To evaluate the central second derivative, we follow the same steps and obtain

$$
\begin{aligned}
\mathcal{R}^{\prime \prime}(0) & =\left.\int \frac{d^{3} k}{(2 \pi)^{3}} \mathcal{R}(k)(\mathrm{i} k)^{2} \exp (\mathrm{ik} \cdot \mathbf{r})\right|_{r=0} \\
& =-\sum_{n=1}^{\infty}\left(a_{0 \mid n}^{0}+\sqrt{\frac{4}{5}} a_{2 \mid n}^{0}\right) \int \frac{d k}{\sqrt{\pi}(2 \pi)^{2}} \psi_{n}(k) k^{4}=\vartheta_{2} .
\end{aligned}
$$

The intermediate steps of the derivation of Eq. (13) are presented in Appendix A.

To proceed with the computation of the probabilities given in Eqs. (5) and (6) we must integrate over all configurations in Fourier space. With the aid of the expansion (8) we can express the measure of such integral in terms of the expansion coefficients satisfying the reality conditions (9) and (10), i.e., for any $\Psi[\mathcal{R}]$, functional of $\mathcal{R}(\mathbf{k})$, the following integral can be represented as

$$
\begin{aligned}
& \int \Psi[\mathcal{R}][\mathrm{d} \mathcal{R}]=\left[\prod_{\ell=0}^{\infty} \prod_{m=1}^{\ell} \prod_{n=1}^{\infty} \mu \int_{-\infty}^{\infty} \Psi[\mathcal{R}] \mathrm{d} a_{\ell \mid n}^{m} \int_{-\infty}^{\infty} \Psi[\mathcal{R}] \mathrm{d} b_{\ell \mid n}^{m}\right] \times \\
& {\left[\prod_{p=0}^{\infty} \prod_{q=1}^{\infty} \tilde{\mu} \int_{-\infty}^{\infty} \Psi[\mathcal{R}] \mathrm{d} a_{2 p \mid q}^{0} \int_{-\infty}^{\infty} \Psi[\mathcal{R}] \mathrm{d} b_{2 p+1 \mid q}^{0}\right] }
\end{aligned}
$$

where the constants $\mu$ and $\tilde{\mu}$ are weight factors. In our calculation of probabilities, such factors are absorbed by the final normalisation of the joint probability.

As mentioned before, we restrict ourselves to the Gaussian PDF. In terms of the spherical harmonic coefficients (see Appendix B), this means that

$$
\mathbb{P}[\mathcal{R}]=\exp \left(-\frac{1}{2 \pi^{2}(2 \pi)^{3}} \sum_{\ell=0}^{\infty} \sum_{m=0}^{\ell} \sum_{n=1}^{\infty}\left|a_{\ell \mid n}^{m}\right|^{2}+\left|b_{\ell \mid n}^{m}\right|^{2}-\frac{1}{4 \pi^{2}(2 \pi)^{3}} \sum_{p=0}^{\infty} \sum_{q=1}^{\infty}\left|a_{2 p \mid q}^{0}\right|^{2}+\left|b_{2 p+1 \mid q}^{0}\right|^{2}\right) .
$$

In order to obtain the probabilities of the mentioned parameters from Eqs. (5) and (6), we use the representation of the Dirac $\delta$-function

$$
\delta(x)=\int_{-\infty}^{\infty} d z \exp [\mathrm{i} z x]
$$

This allows us to write, for example, the $\delta$-function in Eq. (5) in terms of the spherical harmonic coefficients as

$$
\delta\left(\mathcal{R}(0)-\vartheta_{0}\right)=\int d z \exp \left[\mathrm{i} z\left(\sum_{n=1}^{\infty} a_{0 \mid n}^{0} \int_{0}^{\Lambda} d k \psi_{n} k^{2}-\frac{(2 \pi)^{3}}{\sqrt{4 \pi}} \vartheta_{0}\right)\right]
$$


In the same way, the representation of $\delta\left[\mathcal{R}^{\prime \prime}(0)-\vartheta_{2}\right]$ can be written in terms of harmonic coefficients with the aid of Eq. (13).

We now have all the elements needed to derive the probability of the parameters $\mathcal{R}(0)$ and $\mathcal{R}^{\prime \prime}(0)$. Substituting expressions (15) and (17) in Eq. (5), we perform the functional integral with the aid of the decomposition (14). In this process we discard all the Gaussian integrals because they contribute to the probability only with a multiplicative constant which will be included in the final normalisation. On the other hand, the Dirac $\delta$-function contributes with exponential factors of $a_{0 \mid n}^{0}$ to the integrals. The integrals of these parameters are computed by completing squares of the exponential arguments, so the integrals of such coefficients include a set of shifted Gaussian functions (see Appendix B for the details of this procedure). The integral (6) can be performed following the same steps and using the corresponding expressions (13),(14) and (15). The final probability density for the pair of parameters $\mathcal{R}(0)$ and $\mathcal{R}^{\prime \prime}(0)$, is the product of the integrals $(12)$ and (13), i.e.

$$
\mathbb{P}\left(\mathcal{R}(0)=\vartheta_{0}, \mathcal{R}^{\prime \prime}(0)=\vartheta_{2}\right)=A \exp \left(-\frac{\vartheta_{0}^{2}}{2 \Sigma_{(2)}^{2}}-\frac{5 \vartheta_{2}^{2}}{2 \Sigma_{(4)}^{2}}\right)
$$

where $\Sigma_{(2)}$ and $\Sigma_{(4)}$ are the dispersion of the amplitude and the second derivative respectively, and $A$ is a normalisation factor obtained from the condition that the integral of the joint PDF over all possible values of the two independent parameters equals unity. The final normalised joint probability density is

$$
\mathbb{P}\left(\vartheta_{0}, \vartheta_{2}\right)=\frac{4 \sqrt{12}}{2 \pi} \Sigma_{(2)}^{-1} \Sigma_{(4)}^{-1} \exp \left(-\frac{\vartheta_{0}^{2}}{2 \Sigma_{(2)}^{2}}-\frac{5 \vartheta_{2}^{2}}{2 \Sigma_{(4)}^{2}}\right) \text {. }
$$

According to the Press-Schechter formalism of structure formation [4], the PDF is integrated over all perturbations which collapse to form the astrophysical objects under consideration. In this way we calculate the mass fraction of the universe in the form of such objects. To apply this formalism and calculate the probability of PBH formation and integrate the PDF (19), we require the range of values $\mathcal{R}(0)$ and $\mathcal{R}^{\prime \prime}(0)$ which correspond to $\mathrm{PBH}$ formation. In the next section we will obtain this range with the help of the results of numerical computations presented in [18].

\section{THE LINK BETWEEN PERTURBATION PARAMETERS AND THE CURVATURE PROFILES USED IN NUMERICAL CALCULATIONS}

\section{A. Initial conditions}

As demonstrated by the first numerical simulations of $\mathrm{PBH}$ formation [17], whether or not an initial configuration with given curvature profile leads to $\mathrm{PBH}$ formation, predominantly depends on the following two factors:

1) The ratio of the size of the initial configuration $r_{0}$ to the size of the closed universe $r_{\mathrm{k}}=$ $a(t) \int_{0}^{1} d r / \sqrt{1-r^{2}}$ (evaluated at the initial time), which is a measure of the strength of gravitational field within the configuration.

2) The smoothness of the transition from the region of high curvature to the spatially flat FRW universe, which is characterised by the width of the transition region at the edge of the initial configuration and it is inversely proportional to the pressure gradients there. Strong pressure gradients inhibit PBH formation.

The numerical computations presented in [18] (hereafter PM) give the time evolution of the configurations with initial curvature profiles accounting for the above-mentioned factors and collapsing in a radiationdominated universe. In that paper the initial conditions are obtained with the help of the quasi-homogeneous asymptotic solution valid in the limit $t \rightarrow 0$. This solution to the Einstein equations was first introduced by Lifshitz and Khalatnikov [35] (see also [48, 49]). Following [17], PM used this asymptotic solution to set self-consistent initial conditions for curvature inhomogeneities, the initial curvature inhomogeneity being described by the spherically symmetric curvature profile $\mathrm{K}(\hat{r})$. This sets the initial conditions for the process of black hole formation. Asymptotically, the metric can be presented in terms of $\mathrm{K}(\hat{r})$ as

$$
d s^{2}=s^{2}(\eta)\left\{-d \eta^{2}+\frac{1}{1-\mathrm{K}(\hat{r}) \hat{r}^{2}} d \hat{r}^{2}+\hat{r}^{2}\left[d \theta^{2}+\sin ^{2} \theta d \phi^{2}\right]\right\},
$$


where $s(\eta)$ is the scale factor, $\eta$ is the conformal time and we write $\hat{r}$ for the radial coordinate to distinguish it from the coordinate of the metric (1). An advantage of working with this metric is that it contains the curvature profile $\mathrm{K}(\hat{r})$ explicitly. We choose a set of coordinates with the origin at the centre of spherical symmetry and fix $\mathrm{K}(0)=1$. The condition that $\mathrm{K}(\hat{r})$ is a local inhomogeneity requires that $\mathrm{K}(\hat{r})=0$ for radii $\hat{r}$ larger than the scale $\hat{r}_{0}$, where the metric matches the homogeneous FRW background.

In $\mathrm{PM}$ the profiles $\mathrm{K}(\hat{r})$ are presented in two forms, one of which is characterised by two independent parameters $\alpha$ and $\Delta$ as

$$
\mathrm{K}(\hat{r})=\left[1+\alpha \frac{\hat{r}^{2}}{2 \Delta^{2}}\right] \exp \left(-\frac{\hat{r}^{2}}{2 \Delta^{2}}\right)
$$

The results of the numerical simulations in PM indicate that PBHs are formed in the region of the parameter space $[\alpha, \Delta]$ shown in Fig. 1a.
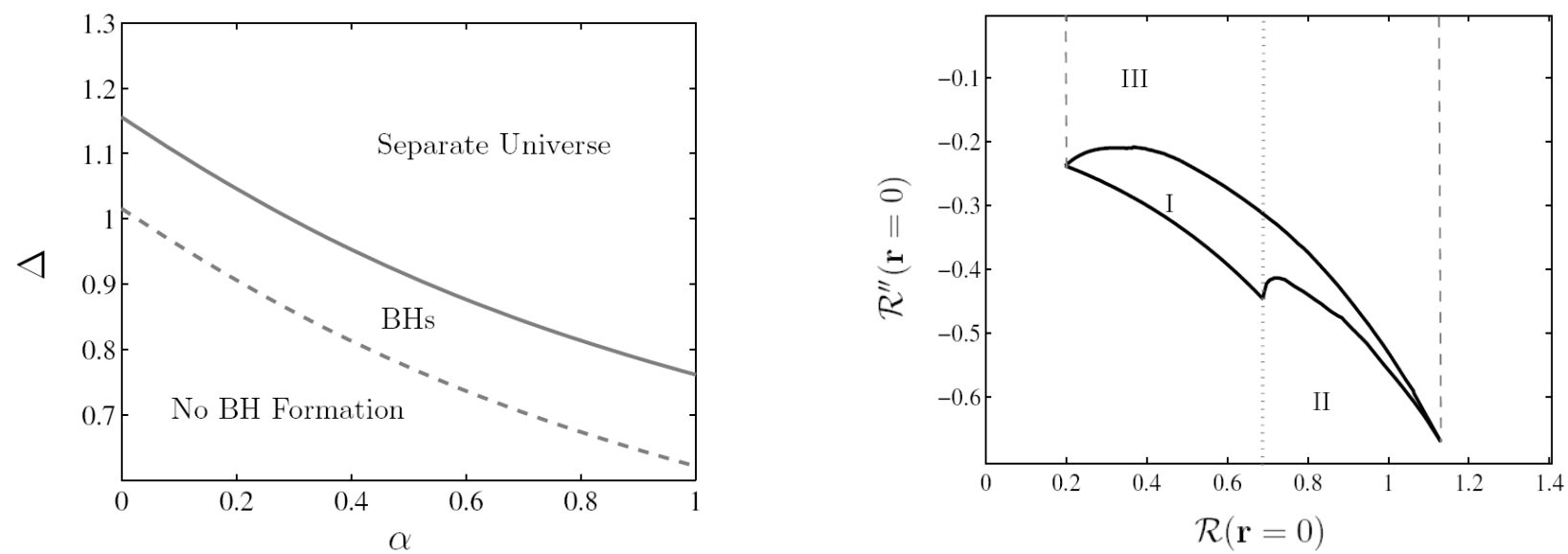

FIG. 1: a) The left plot shows the parameter values for initial configurations which collapse to form black holes. $\Delta$ characterises the width of the Gaussian curvature profile, while $\alpha$ characterises the deviations from a Gaussian profile, as can be seen in Eq. (21). b) In the $\left[\mathcal{R}(0), \mathcal{R}^{\prime \prime}(0)\right]$ plane three regions of integration are considered to compute the probability of $\mathrm{PBH}$ formation. Region I is the region enclosed by the solid curves and corresponds to the region noted by $\mathrm{BH}$ in Fig. 1a. Region II is the region to the right of the grey dotted line representing the surface of integration considered in previous studies where only the amplitude is taken into account [26]. Region III is the region above the solid line and between the dashed lines. The physical characteristics of profiles with values in this region are described in section III C.

\section{B. Physical criteria for the identification of parameters}

We proceed by finding the correspondence between the two sets of parameters, $\left[\mathcal{R}(0), \mathcal{R}^{\prime \prime}(0)\right]$ and $[\alpha, \Delta]$, both of which describe the initial curvature profiles. Assuming that the size of the configuration, $r_{0}$, is much larger than the Hubble horizon $r_{H}=H^{-1}$, where $H$ is the Hubble parameter, we can use the gradient expansion of the functions in metrics (1) and (20). In this case, the time derivative of any function $f(t, r)$ is of order $f / t \sim H f$ and significantly exceeds the spatial gradient which is of order $f / r_{0}$. Hence the small parameter in the gradient expansion is

$$
\epsilon \equiv \frac{r_{H}}{r_{0}}=\frac{k}{a H}
$$

where $k$ is the wave-number corresponding to the scale of the configuration. Taking into account that $\epsilon \rightarrow 0$ when $t \rightarrow 0$, one sees that the gradient expansion is very similar to the quasi-homogeneous solution [35].

For the metric (1), using the coordinate freedom to set $N^{i}=0$ and ignoring any tensor contributions, i.e., 
taking $\tilde{\gamma}_{i j}=\delta_{i j}$, the expansion of the Einstein equation $G_{0}{ }^{0}=8 \pi G T_{0}{ }^{0}$ to order $\epsilon^{2}$ can be written as ${ }^{2}$,

$$
\frac{1}{2}\left(\frac{6 \dot{a}^{2}}{a^{2}}+{ }^{(3)} \mathrm{R}-\frac{4 \dot{a}^{2}}{a^{2}}(N-1)\right)+\mathcal{O}\left(\epsilon^{4}\right)=8 \pi G\left(\rho_{0}+\delta \rho\right)+\mathcal{O}\left(\epsilon^{4}\right),
$$

where ${ }^{(3)} \mathrm{R}$ is the spatial curvature, or the Ricci scalar for the spatial metric $g_{i j}$. To order zero in $\epsilon$, we have

$$
\frac{3 \dot{a}^{2}}{a^{2}}=8 \pi G \rho_{0},
$$

which corresponds to the homogeneous part of (23). As shown in $[16,51,52]$, the time slicing can be set to a uniform expansion gauge in which

$$
N-1=-\frac{3 \Gamma-2}{\Gamma} \delta+\mathcal{O}\left(\epsilon^{4}\right),
$$

where $\Gamma-1$ is the sound-speed squared. Using (23),(24) and (25), we find the equivalence between the spatial curvature and the matter overdensity

$$
{ }^{(3)} \mathrm{R}=\frac{8 \pi G}{3} \delta \rho\left(\frac{4+3 \Gamma}{3 \Gamma}\right) .
$$

In consequence, the gradients establish a correspondence with the pressure gradients

$$
\nabla^{(3)} \mathrm{R}=\frac{8 \pi G}{3} \frac{4+3 \Gamma}{3 \Gamma} \nabla(\delta \rho)=\frac{8 \pi G}{3}\left(\frac{4+3 \Gamma}{3 \Gamma(\Gamma-1)}\right) \nabla p,
$$

where $\nabla=\left(g_{r r}\right)^{-1 / 2} \mathrm{~d} / \mathrm{d} r$. The last equation shows that the gradient of the spatial curvature is directly related to the pressure gradient. Hence, subject to these two physical conditions at the edge of the configuration, we relate the profiles $\mathcal{R}(r)$ and $\mathrm{K}(\hat{r})$ by equating the spatial curvature and its gradient for metrics (1) and (20). That is,

$$
{ }^{(3)} \mathrm{R}=-\left[2 \mathcal{R}^{\prime \prime}(r)+\left(\mathcal{R}^{\prime}(r)\right)^{2}\right] \exp (-2 \mathcal{R}(r))=3 \mathrm{~K}(\hat{r})+\hat{r} \mathrm{~K}^{\prime}(\hat{r}),
$$

and

$$
\begin{gathered}
\frac{1}{\sqrt{g_{r r}}} \frac{\mathrm{d}}{\mathrm{d} r}\left({ }^{(3)} \mathrm{R}\right)= \\
-\left[\mathcal{R}^{\prime} \mathcal{R}^{\prime \prime}+\mathcal{R}^{\prime \prime \prime}\right] \exp (-3 \mathcal{R}(r))=\left[\frac{1-\mathrm{K} \hat{r}^{2}}{\hat{r}^{2}}\right]^{1 / 2}\left(2 \hat{r} \mathrm{~K}^{\prime}(\hat{r})+\frac{1}{2} \hat{r}^{2} \mathrm{~K}^{\prime \prime}(\hat{r})\right) .
\end{gathered}
$$

By definition of the edge of curvature configuration, the three curvature must vanish at this point, so Eq. (28) implies

$$
2 \mathcal{R}^{\prime \prime}\left(r_{0}\right)+\left(\mathcal{R}^{\prime}\left(r_{0}\right)\right)^{2}=0
$$

and

$$
3 \mathrm{~K}\left(\hat{r}_{0}\right)+\hat{r}_{0} \mathrm{~K}^{\prime}\left(\hat{r}_{0}\right)=0 .
$$

As a consequence of this, the gradient relation (29) can be written as

$$
\left[\mathcal{R}^{\prime}\left(r_{0}\right)^{3}-2 \mathcal{R}^{\prime \prime \prime}\left(r_{0}\right)\right] \exp \left(-3 \mathcal{R}\left(r_{0}\right)\right)=\left[\frac{1-\mathrm{K} \hat{r}_{0}^{2}}{\hat{r}_{0}^{2}}\right]^{1 / 2}\left[-12 \mathrm{~K}\left(\hat{r}_{0}\right)+\hat{r}_{0}^{2} \mathrm{~K}^{\prime \prime}\left(\hat{r}_{0}\right)\right] .
$$

\footnotetext{
${ }^{2}$ For the complete second order expansion of the metric quantities, see for example, [29, 32].
} 
This establishes a relation between $\mathcal{R}(r)$ and $\mathrm{K}(\hat{r})$ at the edge points $r_{0}$ and $\hat{r}_{0}$. The configuration $\mathrm{K}(\hat{r})$ is parameterised by $[\alpha, \Delta]$, as shown in Eq. (21). As follows from condition (31) (see also PM), the radius $r_{0}$ can be written in terms of those parameters as

$$
\hat{r}_{0}^{2}=\left(\frac{5 \alpha-2+\sqrt{(5 \alpha-2)^{2}-24 \alpha}}{2 \alpha}\right) \Delta^{2} .
$$

Then we use two more equations obtained from the conformal transformation of coordinates at zero order in $\epsilon$ :

$$
a^{2}(\tau) \mathrm{e}^{2 \mathcal{R}(r)} d r^{2}=s^{2}(\eta) \frac{d \hat{r}^{2}}{1-\mathrm{K}(\hat{r}) \hat{r}^{2}}
$$

and

$$
a^{2}(\tau) \mathrm{e}^{2 \mathcal{R}(r)} r^{2} d \Omega^{2}=s^{2}(\eta) \hat{r}^{2} d \Omega^{2} .
$$

Because the homogeneous Einstein equations are identical in both metrics, the scale factors $a(\tau)$ and $s(\eta)$ can be identified, $a(\tau) \equiv s(\eta)$. Thus we find a relation between the radial coordinates,

$$
\mathrm{e}^{\mathcal{R}(r)} r=\hat{r}
$$

and an integral relation between the configurations

$$
\int_{0}^{r} \mathrm{e}^{\mathcal{R}(x)} d x=\int_{0}^{\hat{r}} \frac{d x}{\sqrt{1-\mathrm{K}(x) x^{2}}}
$$

One can verify that Eqs. (28), (29) and (37) are not independent. For example, Eq. (29) follows from (28) and (37).

In the previous section we have developed a method to account for the probability of any set of parameters describing the curvature profile. For simplicity we have chosen the pair $\left[\mathcal{R}(0), \mathcal{R}^{\prime \prime}(0)\right]$. We now illustrate how to relate $\left[\mathcal{R}(0), \mathcal{R}^{\prime \prime}(0)\right]$ and $[\alpha, \Delta]$ by considering the parabolic profiles

$$
\mathcal{R}(r)=\mathcal{R}(0)+\frac{1}{2} \mathcal{R}^{\prime \prime}(0) r^{2} .
$$

This parametrisation meets the minimal requirement of covering the $[\alpha, \Delta]$ parameter space in Fig. 1a. Eqs. (30), (37) and (36) are now reduced to the following system of algebraic equations:

$$
\begin{gathered}
r_{0}^{2}=-\frac{2}{\mathcal{R}^{\prime \prime}(0)}, \\
\mathcal{R}(0)=2 \log \left(\frac{2}{\operatorname{erf}(1)}\left[\pi \exp (1) \hat{r}_{0}\right]^{-1 / 2} \int_{0}^{\hat{r}_{0}} \frac{d x}{\left(1-\mathrm{K}(x) x^{2}\right)^{1 / 2}}\right), \\
\mathcal{R}^{\prime \prime}(0)=-2 \frac{\exp (2 \mathcal{R}(0)-2)}{\hat{r}_{0}^{2}},
\end{gathered}
$$

where $\hat{r}_{0}$ is given in terms of $[\alpha, \Delta]$ by Eq. (33).

\section{Parameter values leading to PBH formation}

As follows from the numerical computations [18] which used the parametrisation (21), PBHs are formed in the $[\alpha, \Delta]$ region shown in Fig. 1a. Equations (40) and (41) map this region to the Region I in the space of parameters $\left[\mathcal{R}(0), \mathcal{R}^{\prime \prime}(0)\right]$ shown in Fig. 1b. The Jacobian of the transformation corresponding to this mapping is non-vanishing, which guarantees a one-to-one correspondence of the region 'BHs' plotted in Fig. 1a with Region I in Fig. 1b. Each point here corresponds to a parabolic profile which leads to the formation of PBH.

For each one of these parabolic profiles, there is a family of non-parabolic profiles with the same central amplitude $\mathcal{R}(0)$, the same configuration size $r_{0}$, and the same behaviour near the edge, as shown in Fig. 2. 


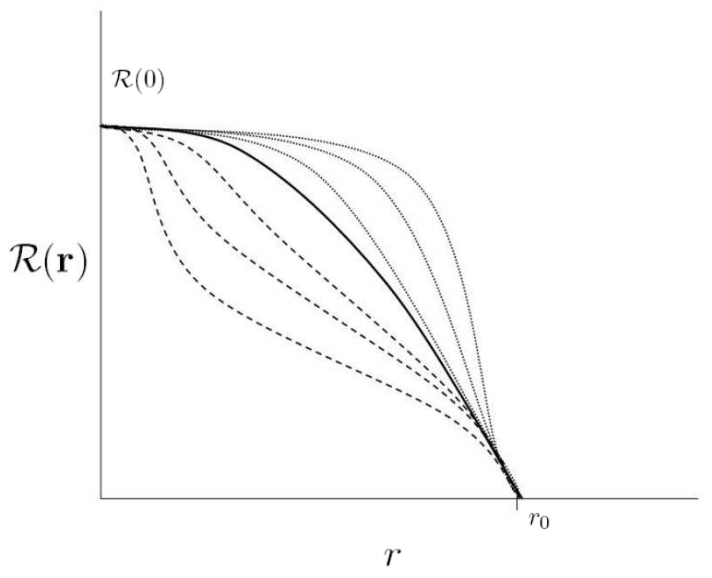

FIG. 2: The curvature profile for three different families of configurations with common central amplitude $\mathcal{R}(0)=1$. The configurations shown by the dashed lines have value of $\mathcal{R}^{\prime \prime}(0)$ larger in absolute magnitude than the parabolic one shown in black. The configurations shown by the dotted lines have a value of $\mathcal{R}^{\prime \prime}(0)$ smaller than the parabolic one. All profiles satisfy conditions (30) and (32).
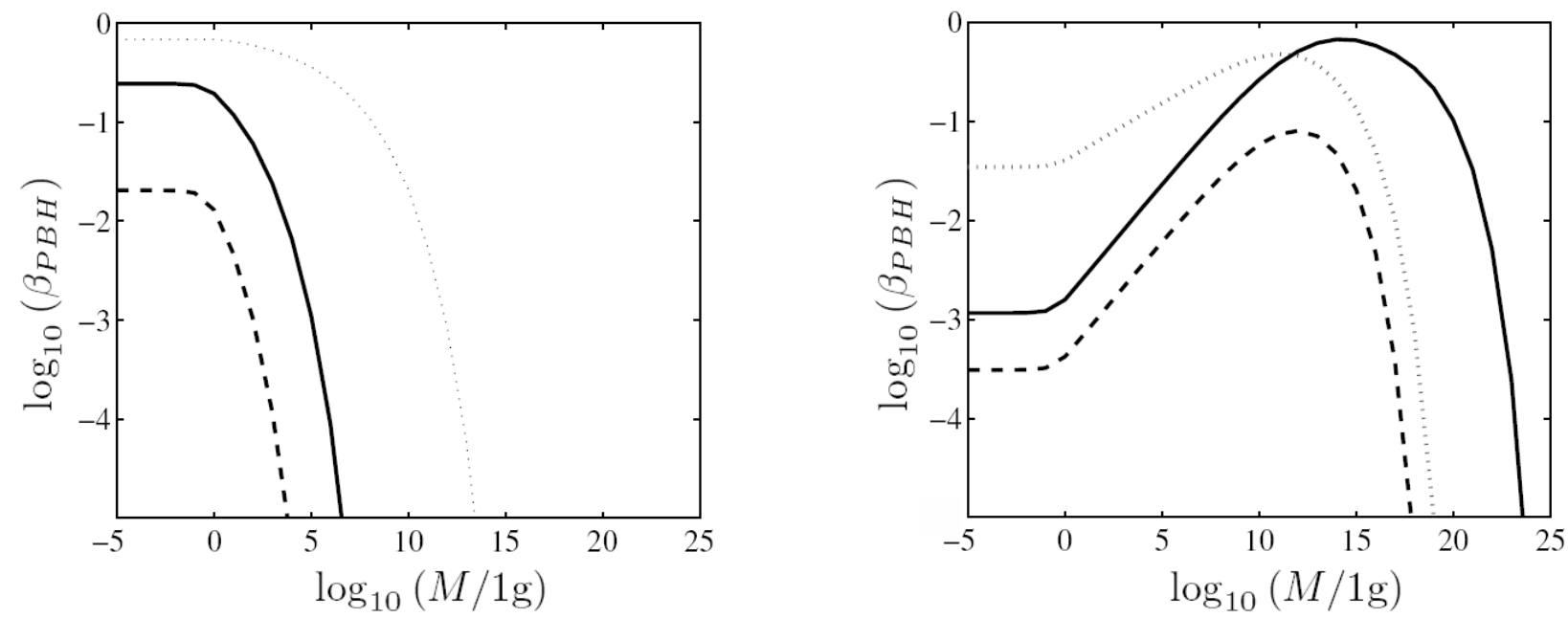

FIG. 3: The logarithmic probability of PBHs $\beta$ calculated using Eq. (42) with a power spectrum with two tilt values $\left(n_{s}=1.32\right.$ for the left plot, $n_{s}=1.47$ for the right plot). The lines show the integration for the three different regions sketched in Fig. 1. The integral over the region I $\left(\beta_{\mathrm{I}}(M)\right)$ corresponds to the dashed lines, and the integration over the region II $\left(\beta_{\mathrm{II}}(M)\right)$ to the solid lines. The probability integrated over the region III $\left(\beta_{\mathrm{III}}(M)\right)$ is represented by the dotted lines in both figures.

In that figure, the profiles lying below the parabola correspond to larger absolute magnitudes of $\mathcal{R}^{\prime \prime}(0)$ and do not form PBHs because they have lower average gravitational field strength and higher average pressure gradient. The non-parabolic profiles which lie above the parabolic one (with smaller absolute magnitude $\mathcal{R}^{\prime \prime}(0)$ ) should also collapse to form PBHs because they correspond to higher average gravitational field strength and lower pressure gradient.

In the parameter-space $\left[\mathcal{R}(0), \mathcal{R}^{\prime \prime}(0)\right]$, this last set of profiles corresponds to Region III in Fig. 1b. This region will be included in the calculation of the probability of $\mathrm{PBH}$ formation in the next section. 


\section{TWO PARAMETRIC PROBABILITY OF PBH FORMATION}

To calculate the probability of $\mathrm{PBH}$ formation, which is equivalent to the mass fraction of the universe going to PBHs of given mass, it is customary to use the standard Press-Schechter formalism [4]. This has been widely used in previous calculations of the one parametric probability of $\mathrm{PBH}$ formation $[3,5,6,8,53,54]$. When the probability depends on a single amplitude parameter, this method reduces to the integration of the corresponding PDF over the relevant perturbation amplitudes. The final integral is equivalent to the mass fraction of PBHs of mass $M \sim(\Gamma-1)^{3 / 2} M_{H} \approx(\Gamma-1)^{3 / 2} k_{M} /(2 \pi)$ [3], with the soundspeed $\sqrt{\Gamma-1}$ measured at the time formation ${ }^{3}$. Here we extend the standard Press-Schechter formalism to derive a two parametric probability, introducing the second derivative at the centre of the configuration as an additional parameter. When the $\left[\mathcal{R}^{\prime}(0), \mathcal{R}^{\prime \prime}(0)\right]$ region is a square $\left[\mathcal{R}_{1}<\mathcal{R}(0)<\mathcal{R}_{2}, \mathcal{R}_{1}^{\prime \prime}<\mathcal{R}^{\prime \prime}(0)<\mathcal{R}_{2}^{\prime \prime}\right]$, the integrated two parametric probability for objects of mass $M$ is

$$
\begin{aligned}
\beta_{P B H}(M)=\int_{\mathcal{R}_{1}}^{\mathcal{R}_{2}} d \vartheta_{0} \int_{\mathcal{R}_{1}^{\prime \prime}}^{\mathcal{R}_{2}^{\prime \prime}} d \vartheta_{2} \mathbb{P}\left(\vartheta_{0}, \vartheta_{2}\right)= \\
\frac{1}{2}\left[\operatorname{erf}\left(\frac{\mathcal{R}_{2}}{\sqrt{2} \Sigma_{(2)}(M)}\right)-\operatorname{erf}\left(\frac{\mathcal{R}_{1}}{\sqrt{2} \Sigma_{(2)}(M)}\right)\right] \times\left[\operatorname{erf}\left(\frac{\mathcal{R}_{2}^{\prime \prime}}{\sqrt{2} \Sigma_{(4)}(M)}\right)-\operatorname{erf}\left(\frac{\mathcal{R}_{1}^{\prime \prime}}{\sqrt{2} \Sigma_{(4)}(M)}\right)\right] .
\end{aligned}
$$

We use this result to integrate numerically over a mesh of small squares covering each one of the regions of the plane $\left[\mathcal{R}(0), \mathcal{R}^{\prime \prime}(0)\right]$ shown in Fig. 1b. We call the integral over region I $\beta_{\mathrm{I}}(M)$, and correspondingly the integrals over regions II and III are called $\beta_{\mathrm{II}}(M)$ and $\beta_{\mathrm{III}}(M)$. The mass dependence of these betas for two different power-law spectra $\mathcal{P}_{\mathcal{R}}(k) \propto k^{n-1}$, are shown in Fig. 3. As dictated by the Press-Shechter formulation, such integration corresponds to the fraction of mass density in the universe that has collapsed into objects with mass $M$. We remind the reader that our choice of $n_{s}$ and the mean amplitude is for pure illustration purposes. With the values used in this paper, copious amounts of black holes are produced. A red spectral index and power spectrum inferred from the CMB data corresponds to a low number of PBHs. However, to assume that the same values of the power spectrum and spectral index are valid on scales relevant to PBH formation (30 decades of mass below the mass scales correspondent to CMB observations) is a very strong extrapolation. At the present time we cannot exclude that the values of $n_{s}$ and the power spectrum are different than those given by the CMB. To explore the structure formation models that match CMB observation values and also produce considerable number of PBHs is a great task beyond the scope of our paper. This important issue is currently under investigation $[10,55]$.

We contrast the case of parabolic profiles described by Eq. (38) with the non-parabolic set presented in Fig. 2 by plotting the ratios of probabilities $\beta_{\mathrm{I}} / \beta_{\mathrm{II}}$ and $\beta_{\mathrm{III}} / \beta_{\mathrm{I}}$ for different values of $\mathcal{P}_{\mathcal{R}}$. This is presented in Fig. 4. This figure shows that the probability of PBH formation can be larger than the previous oneparameter probability computed from the integration of Region II as done in previous studies [26]. This important result requires confirmation from more detailed numerical simulations of $\mathrm{PBH}$ formation in this region of parameter-space. The uncertainty is explained by the fact that the two parametric calculation of the probability of $\mathrm{PBH}$ formation is still incomplete. This should be complemented in the future by the introduction of all relevant higher derivative parameters and the higher-order correlations in the PDF.

\section{DISCUSSION}

We have developed a method for calculating the two-parametric probability of $\mathrm{PBH}$ formation, taking into account the radial profiles of non-linear curvature cosmological inhomogeneities. This is the fist step towards calculating the $N$-parametric probability, which takes into account the radial profiles more precisely than studies using the amplitude as the only relevant parameter. Using the results of sophisticated numerical computations, we obtain the values of $\mathcal{R}^{\prime \prime}(0)$ that are relevant for PBH formation. Subsequently we have incorporated these values to the total probability of PBH formation. Finally, we have provided an example of the consequences of this probability for the statistics of PBHs.

\footnotetext{
3 Throughout this paper we consider configurations that collapse in a uniform radiation dominated background. Thus we use the value $\Gamma=4 / 3$
} 


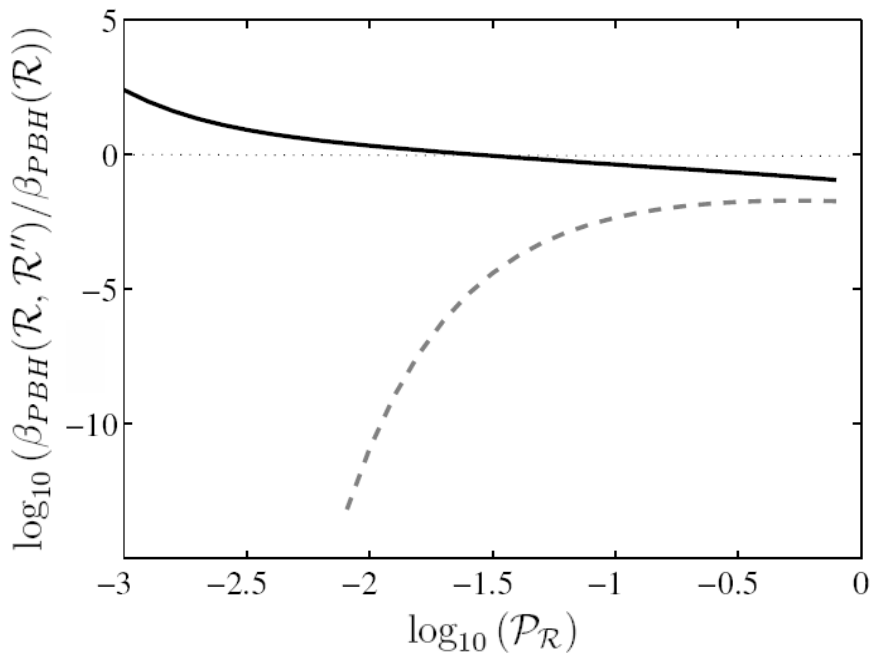

FIG. 4: The horizontal axis of the figure is the amplitude of the power spectrum at scales relevant to PBH formation. The grey dashed line shows the ratio $\beta_{\mathrm{I}} / \beta_{\mathrm{II}}$ where $\beta_{\mathrm{I}}$ is the probability density integrated over region $\mathrm{I}$ in the $\left[\mathcal{R}(0), \mathcal{R}^{\prime \prime}(0)\right]$-parameter space of Fig. $1 \mathrm{~b}$, and $\beta_{\text {II }}$ is the corresponding probability integrated over region II of the same figure. The black line is the ratio $\beta_{\mathrm{III}}$ over $\beta_{\mathrm{II}}$. The dotted line shows the reference case of the single-parameter probability.

The results obtained show that, if we restrict ourselves to the PBH formation calculated for parabolic profiles only (as described in Section III), then the total probability of PBH formation is orders of magnitude below previous estimations. On the other hand, with the aid of heuristic arguments we show that a much larger region of parameter-space $\left[\mathcal{R}(0), \mathcal{R}^{\prime \prime}(0)\right]$ representing non-parabolic profiles should also be considered in the estimation of the probability of $\mathrm{PBH}$ formation (see Fig. 2). In this case, the total probability of $\mathrm{PBH}$ formation is higher than the single-parameter estimate of previous works. In this case, we have an opportunity to impose new bounds on the power spectrum on the scales relevant for PBH formation. Analysing the uncertainty of our results, mostly due to the heuristic nature of the present study, we have demonstrated how much we still have to understand about the formation and statistics of PBHs. The physical arguments supporting our results should be made rigorous by direct verification with numerical hydrodynamical simulations of $\mathrm{PBH}$ formation. This in turn would provide valuable support for the initial motivation of this work.

The main conclusion of this paper is that the amplitude of initial inhomogeneities is not the only parameter which determines the probability of $\mathrm{PBH}$ formation. The ultimate solution of the problem requires a greater set of parameters and a larger range of their values to determine all high curvature configurations that form PBHs, which is a huge task for future research. In the meantime, we have a method to operate with the statistics of all these parameters.

\section{ACKNOWLEDGEMENTS}

JCH gratefully acknowledges financial support from the Mexican Council for Science and Technology (CONACYT) (Studentship No. 179026). We would like to thank Prof Bernard Carr, Dr Karim Malik, Dr David Seery and Dr Ilia Musco for useful comments and discussions. 
APPENDIX A: HARMONIC DECOMPOSITION OF $\mathcal{R}$ AND FOURIER REPRESENTATION OF $\mathcal{R}(0)$ AND $\mathcal{R}^{\prime \prime}(0)$

The Fourier expansion of the smoothed curvature perturbation profile $\mathcal{R}$ in terms of spherical harmonic functions is,

$$
\mathcal{R}(\mathbf{k}, t)=\sum_{\ell=0}^{\infty} \sum_{m=-\ell}^{\ell} \sum_{n=1}^{\infty} \mathcal{R}_{\ell \mid n}^{m}(t) Y_{\ell m}\left(\theta_{k}, \phi_{k}\right) \psi_{n}(k)
$$

The radial functions of the harmonic decomposition can be defined by

$$
\psi_{n}(k)=\frac{\sqrt{2}}{J_{\nu+1}\left(\alpha_{\nu}^{n}\right)} \frac{\mathcal{P}(k) \mathcal{W}\left(k ; k_{\mathrm{M}}\right)}{\Lambda k^{2}} J_{\nu}\left(\alpha_{\nu}^{n} \frac{k}{\Lambda}\right),
$$

where $\mathcal{W}\left(k ; k_{\mathrm{M}}\right)$ is the window function with smoothing scale $k_{\mathrm{M}}$ and $\alpha_{\nu}^{n}$ is the $n$-th root of the Bessel function of order $\nu, J_{\nu}(k)$. Note that the functions of the radial coordinate in the expansion are the Bessel functions up to a factor. The set of functions $\psi_{n}(k)$ is orthonormal under the product

$$
\int_{0}^{\Lambda} \mathrm{d} k \frac{k^{5}}{\mathcal{P}(k) \mathcal{W}^{2}\left(k ; k_{\mathrm{M}}\right)} \psi_{m}(k) \psi_{n}(k)=\delta_{m n},
$$

and the completeness relation can be written with the aid of the Kernel in the internal product:

$$
\frac{k_{0}^{5}}{\mathcal{P}\left(k_{0}\right) \mathcal{W}^{2}\left(k_{\mathrm{M}}\right)} \sum_{n} \psi_{n}\left(k_{0}\right) \psi_{n}(k)=\delta\left(k-k_{0}\right) .
$$

In Eq. (A3) $\Lambda$ represents an artificial compactification of the momentum space which is used only to have an explicit definition of $\psi$ at hand. With the definitions above, the expansion (A1) describes the curvature perturbation with power spectrum $\mathcal{P}(k)$ smoothed over a scale $k_{\mathrm{M}}$.

Let us now construct a parameter to represent the second radial derivative of the field $\mathcal{R}$ in Fourier space. The first radial derivative of $\mathcal{R}(\mathbf{r})$ is

$$
\frac{\partial}{\partial r} \mathcal{R}(\mathbf{r})=\int d^{3} k \sum_{l, m, n} \mathcal{R}_{l, n}^{m} Y_{l}^{m}\left(\theta_{k}, \phi_{k}\right) \psi_{n}(k) \mathrm{e}^{\mathrm{i}(\mathbf{k} \cdot \mathbf{r})} \times \frac{\partial}{\partial r}(\mathrm{ik} \cdot \mathbf{r}) .
$$

If we write the spherical coordinates in a Cartesian basis, we have

$$
\mathbf{k} \cdot \mathbf{r}=|k||r|\left\{\sin \left(\theta_{r}\right) \cos \left(\phi_{r}\right) \sin \left(\theta_{k}\right) \cos \left(\phi_{k}\right)+\sin \left(\theta_{r}\right) \sin \left(\phi_{r}\right) \sin \left(\theta_{k}\right) \sin \left(\phi_{k}\right)+\cos \left(\theta_{r}\right) \cos \left(\theta_{k}\right)\right\},
$$

with $\left(\phi_{r}, \theta_{r}\right)$ the set of angles of the vector $\mathbf{r}$ and $\left(\phi_{k}, \theta_{k}\right)$ the corresponding pair for $\mathbf{k}$.

The scalar product (A6) can be simplified if we note that the integral in Fourier space spans all possible directions of $\mathbf{k}$, so we can choose an arbitrary direction for $\mathbf{r}$. In particular, we can fix the $\mathbf{r}$ so that $\cos \left(\theta_{r}\right)=1$. This allows us to write a simple expression for the radial derivative,

$$
\frac{\partial}{\partial r}(\mathbf{k} \cdot \mathbf{r})=|k| \cos \left(\theta_{k}\right)
$$

The first derivative of the profile $\mathcal{R}(\mathbf{r})$ at the centre of the configuration is zero by the symmetry of spherical configurations. By construction of the spherically symmetric Fourier modes, this condition is satisfied identically at $\mathbf{r}=0$. The first non-vanishing parameter that gives information about of the profile of perturbations is the second derivative. Fixing the direction of the vector $\mathbf{r}$ in the scalar product we have

$$
\frac{\partial^{2}}{\partial r^{2}} \mathcal{R}(\mathbf{r})=\int d^{3} k \sum_{l, m, n} \mathcal{R}_{l, n}^{m} Y_{l}^{m}\left(\theta_{k}, \phi_{k}\right) \psi_{n}(k) \times k^{2} \cos ^{2}\left(\theta_{k}\right) \mathrm{e}^{\mathrm{i}(\mathbf{k} \cdot \mathbf{r})}
$$

With the standard definition of the spherical harmonics,

$$
Y_{l}^{m}(\theta, \phi)=\sqrt{\frac{2 l+1}{4 \pi} \frac{(l-m) !}{(l+m) !}} P_{l}^{m}(\cos (\theta)) \mathrm{e}^{\mathrm{i} m \phi},
$$


where the normalisation factor is used for orthonormality purposes, one can write the factor $\cos ^{2}\left(\theta_{k}\right)$ as the sum of two spherical harmonics,

$$
\cos ^{2}\left(\theta_{k}\right)=\frac{1}{3} \sqrt{4 \pi}\left(\sqrt{\frac{4}{5}} Y_{2}^{0}+Y_{0}^{0}\right)=\frac{1}{3} \sqrt{4 \pi}\left(\sqrt{\frac{4}{5}} \bar{Y}_{2}^{0}+\bar{Y}_{0}^{0}\right),
$$

where the $\bar{Y}$ indicates the complex conjugate.

Using the normalisation rule for spherical harmonics,

$$
\int d \Omega Y_{l}^{m}(\theta, \phi) \bar{Y}_{k}^{n}(\theta, \phi)=\delta_{m n} \delta_{l k},
$$

we can integrate Eq. (A10) in the derivative (A8) and arrive at the expression

$$
\mathcal{R}^{\prime \prime}(0)=\int d k k^{4}\left(\frac{\sqrt{4 \pi}}{3}\right)\left[\sum_{n=1}^{\infty}\left(\sqrt{\frac{4}{5}} \mathcal{R}_{2, n}^{0}+\mathcal{R}_{0, n}^{0}\right) \psi_{n}\right] .
$$

This is the result used in Section II.

To finish this appendix we show how the introduction of a parameter off the centre, say $\mathcal{R}^{\prime}\left(r_{0}\right)$, generates a large set of constraints on the values of the coefficients $\mathcal{R}_{\ell \mid n}^{0}$. The integral in Fourier space representing this derivative is

$$
\begin{aligned}
\left.\mathcal{R}^{\prime}(r)\right|_{r=r_{0}} & =\mathrm{i} \int \frac{d^{3} k}{(2 \pi)^{3}}|k| \mathcal{R}(\mathbf{k}) \cos \left(\theta_{k}\right) \exp \left[\mathrm{i} k r \cos \left(\theta_{k}\right)\right] \\
& =\mathrm{i} \int \frac{d^{3} k}{(2 \pi)^{3}}|k| \mathcal{R}(\mathbf{k}) Y_{1}^{0}(\theta, \phi)\left[\sum_{s=0}^{\infty} \frac{\left(\mathrm{i} \cos \left(\theta_{k}\right) r_{0} k\right)^{s}}{s !}\right]
\end{aligned}
$$

where we have expanded the exponential function in Taylor series. Each power of $\cos (\theta)$ can be expressed in terms of spherical harmonic functions $Y_{\ell}^{0}$. This means that the last integral consists of a series of terms of the form,

$$
\int d \Omega Y_{\ell}^{n}(\theta, \phi) Y_{1}^{0}(\theta, \phi) Y_{S}^{0}(\theta, \phi) .
$$

Each of these integrals is a Clebsch-Gordan coefficient. These steps are enough to show that, while a parameter $\mathcal{R}^{\prime}\left(r=r_{0}\right)$ might represent an improvement in the estimate of the final probability of PBH formation, it takes a long calculation to complete squares, add normalisation factors for each coefficient $\mathcal{R}_{\ell \mid n}^{0}$, and arrive at a final expression like eq. (19). This goes beyond the goals of the present paper.

\section{APPENDIX B: THE PROBABILITY OF $\mathcal{R}(0)$ AND $\mathcal{R}^{\prime \prime}(0)$}

At any time $t$, the probability distribution $\mathbb{P}_{t}[\mathrm{R}]$, is formally obtained through the inverse Fourier transform of $Z_{t}[\eta]$, a generating functional which can be expanded in terms of the $n$-point correlation functions [36],

$$
Z_{t}[\eta]=\exp \sum_{n=0}^{\infty} \frac{\mathrm{i}^{n}}{n !} \int \cdots \int \mathrm{d}^{3} y_{1} \cdots \mathrm{d}^{3} y_{n} \eta\left(\mathbf{y}_{1}\right) \cdots \eta\left(\mathbf{y}_{n}\right)\left\langle\mathcal{R}\left(t, \mathbf{y}_{1}\right) \cdots \mathcal{R}\left(t, \mathbf{y}_{n}\right)\right\rangle
$$

Hence, up to an overall normalisation,

$$
\mathbb{P}_{t}[\mathcal{R}] \propto \int[\mathrm{d} \eta] \exp \left(-\mathrm{i} \int \mathrm{d}^{3} \mathrm{x} \mathcal{R}(\mathbf{x}) \eta(\mathbf{x})\right) Z_{t}[\eta] .
$$

If all correlation functions of three and more points are set to zero, then $\mathbb{P}[\mathcal{R}] \propto \mathbb{G}[\mathcal{R}]$. Assuming this for the generating functional (B1), the expression to integrate in the Fourier space is,

$$
\mathbb{P}_{t}[\eta ; \mathcal{R}]=\exp \left(-\int \frac{\mathrm{d}^{3} k_{1} \mathrm{~d}^{3} k_{2}}{(2 \pi)^{6}} \frac{\eta\left(\mathbf{k}_{1}\right) \eta\left(\mathbf{k}_{2}\right)}{2}\left\langle\mathcal{R}\left(t, \mathbf{k}_{1}\right) \mathcal{R}\left(t, \mathbf{k}_{2}\right)\right\rangle-\mathrm{i} \int \frac{\mathrm{d}^{3} k}{(2 \pi)^{3}} \eta(\mathbf{k}) \mathcal{R}(\mathbf{k})\right) .
$$


The functional integral of this expression gives the probability of $\mathcal{R}$. To solve this integral we complete the square of $\eta$ factors and make the finite field redefinition

$$
\eta(\mathbf{k}) \mapsto \hat{\eta}(\mathbf{k})=\eta(\mathbf{k})+\mathrm{i}(2 \pi)^{3} \frac{\mathrm{R}(\mathbf{k})}{\langle\mathcal{R}(t, \mathbf{k}) \mathcal{R}(t,-\mathbf{k})\rangle^{\prime}},
$$

where the prime ' attached to $\langle\mathcal{R}(t, \mathbf{k}) \mathcal{R}(t,-\mathbf{k})\rangle^{\prime}$ indicates that the momentum-conservation $\delta$-function is omitted. The measure $[\mathrm{d} \eta]$ is invariant under this shift, giving $\int[\mathrm{d} \eta]=\int[\mathrm{d} \hat{\eta}]$, whereas $\mathbb{P}_{t}[\eta ; \mathcal{R}]$ can be split into an $\mathcal{R}$-dependent piece, which we call $\mathbb{G}_{t}[\mathcal{R}]$, and a piece that depends only on $\hat{\eta}$ but not $\mathcal{R}$,

$$
\mathbb{P}_{t}[\eta ; \mathcal{R}] \mapsto \mathbb{G}_{t}[\mathcal{R}] \exp \left(-\frac{1}{2} \int \frac{\mathrm{d}^{3} k_{1} \mathrm{~d}^{3} k_{2}}{(2 \pi)^{6}} \hat{\eta}\left(\mathbf{k}_{1}\right) \hat{\eta}\left(\mathbf{k}_{2}\right)\left\langle\mathcal{R}\left(t, \mathbf{k}_{1}\right) \mathcal{R}\left(t, \mathbf{k}_{2}\right)\right\rangle\right),
$$

where $\mathbb{G}_{t}[\mathcal{R}]$ is a Gaussian in $\mathcal{R}$,

$$
\mathbb{G}_{t}[\mathcal{R}]=\exp \left(-\frac{1}{2} \int \mathrm{d}^{3} k_{1} \mathrm{~d}^{3} k_{2}\left\langle\mathcal{R}\left(t, \mathbf{k}_{1}\right) \mathcal{R}\left(t, \mathbf{k}_{2}\right)\right\rangle \frac{\mathcal{R}\left(\mathbf{k}_{1}\right) \mathcal{R}\left(\mathbf{k}_{2}\right)}{\prod_{i}\left\langle\mathcal{R}\left(t, \mathbf{k}_{i}\right) \mathcal{R}\left(t,-\mathbf{k}_{i}\right)\right\rangle^{\prime}}\right) .
$$

When we make the expansion of the fields $\mathcal{R}(\mathbf{k})$ in terms of the spherical harmonics as in Eq. (A1) and using the explicit expression for the two point correlation Eq. (3) we obtain,

$$
\begin{array}{r}
\mathbb{G}[\mathcal{R}]=\exp \left(-\frac{1}{2} \int \mathrm{d} \Omega \int k^{2} \mathrm{~d} k \frac{k^{3}}{(2 \pi)^{3} 2 \pi^{2}} \frac{1}{\mathcal{P}(k) \mathcal{W}^{2}(k)}\right. \\
\left.\times \sum_{\ell_{1}, m_{1}, n_{1}} \sum_{\ell_{2}, m_{2}, n_{2}} \mathcal{R}_{\ell_{1} \mid n_{1}}^{m_{1}} \mathcal{R}_{\ell_{2} \mid n_{2}}^{m_{2} \dagger} Y_{\ell_{1} m_{1}}(\theta, \phi) Y_{\ell_{2}, m_{2}}^{\dagger}(\theta, \phi) \psi_{n_{1}}(k) \psi_{n_{2}}(k)\right),
\end{array}
$$

where the normalisation factor has been left aside and can be recovered by demanding the integral over all values to be equal to 1 .

The harmonics $Y_{\ell m}$ and $\psi_{n}$ integrate out of this expression entirely, using the orthonormality relation (A3) and the spherical harmonic completeness relation (A11). Moreover, after rewriting the $a$ and $b$ coefficients with $m<0$ in terms of the $m>0$ coefficients, we obtain

$$
\mathbb{G}[\mathcal{R}]=\exp \left(-\frac{1}{2 \pi^{2}(2 \pi)^{3}} \sum_{\ell=0}^{\infty} \sum_{m=1}^{\ell} \sum_{n=1}^{\infty}\left|a_{\ell \mid n}^{m}\right|^{2}+\left|b_{\ell \mid n}^{m}\right|^{2}-\frac{1}{4 \pi^{2}(2 \pi)^{3}} \sum_{\substack{\ell=0 \\ \ell \text { even }}}^{\infty} \sum_{n=1}^{\infty}\left|a_{\ell \mid n}^{0}\right|^{2}+\left|b_{\ell+1 \mid n}^{0}\right|^{2}\right) .
$$

which is the gaussian expression presented in (15).

In order to find the probability for given values of the central amplitude $\mathcal{R}(r=0)=\vartheta_{0}$, we integrate $\mathbb{G}[\mathcal{R}]$ with the $\delta$-function factor in (17). We introduce the Fourier representation of the $\delta$-function to write,

$$
\mathbb{P}\left(\vartheta_{0}\right) \propto \int[\mathrm{d} \mathcal{R}] \int_{-\infty}^{\infty} \mathrm{d} z \mathbb{G}[\mathcal{R}] \exp \left[\mathrm{i} z\left(\sum_{n=1}^{\infty} a_{0 \mid n}^{0} \Sigma_{n}-\frac{(2 \pi)^{3}}{\sqrt{4 \pi}} \vartheta_{0}\right)\right]
$$

where the functional measure is understood to be Eq. (14). The final answer is obtained by integrating out $z$ together with all of the $a$ and $b$ coefficients. In order to achieve this, it is necessary to decouple $a_{0 \mid n}^{0}, z$ and $\vartheta_{0}$ from each other by successively completing the square in $a_{0 \mid 0}^{0}$ and $z$. Working with $a_{0 \mid 0}^{0}$ first, we find

$$
\begin{aligned}
& \exp \left(-\frac{1}{4 \pi^{2}} \frac{1}{(2 \pi)^{3}} \sum_{n=1}^{\infty}\left|a_{0 \mid n}^{0}\right|^{2}+\mathrm{i} z \sum_{n=1}^{\infty} a_{0 \mid n}^{0} \Sigma_{n}\right) \\
= & \exp \left[-\frac{1}{4 \pi^{2}} \frac{1}{(2 \pi)^{3}} \sum_{n=1}^{\infty}\left(a_{0 \mid n}^{0}-\mathrm{i} 2 \pi^{2}(2 \pi)^{3} z \Sigma_{n}\right)^{2}-(2 \pi)^{3} \pi^{2} z^{2} \Sigma_{(2)}^{2}\right],
\end{aligned}
$$

where we have introduced a function $\Sigma_{(2)}^{2}$, defined by $\Sigma_{(2)}^{2}=\sum_{n=1}^{\infty} \Sigma_{n}^{2}$. In the final probability distribution, $\Sigma_{(2)}^{2}$ will turn out to be the variance of $\mathcal{R}(0)$. From Eq. (B10), it is clear that making the transformation 
$a_{0 \mid n}^{0} \mapsto a_{0 \mid n}^{0}+\mathrm{i} 2 \pi^{2}(2 \pi)^{3} z \Sigma_{n}$ suffices to decouple $a_{0 \mid n}^{0}$ from $z$. The measure, Eq. (14), is formally invariant under this transformation. We can also complete squares for the variables $z$ and $\vartheta_{0}$, giving

$$
\exp \left(-(2 \pi)^{3} \pi^{2} z^{2} \Sigma_{(2)}^{2}-\mathrm{i} \frac{(2 \pi)^{3}}{\sqrt{4 \pi}} \vartheta_{0} z\right)=\exp \left[-(2 \pi)^{3} \pi^{2} \Sigma_{(2)}^{2}\left(z+\mathrm{i} \frac{\vartheta_{0}}{2 \pi^{2} \sqrt{4 \pi} \Sigma_{(2)}^{2}}\right)^{2}-\frac{\vartheta_{0}^{2}}{2 \Sigma^{2}}\right]
$$

As before, the finite shift $z \mapsto z-\mathrm{i} \vartheta_{0} / 2 \pi^{2} \sqrt{4 \pi} \Sigma_{(2)}^{2}$ leaves the measure intact and decouples $z$ and $\vartheta_{0}$. The $a, b$ and $z$ integrals can be done independently, but since they do not involve $\vartheta_{0}$ they contribute only an irrelevant normalisation to $\mathbb{P}\left(\vartheta_{0}\right)$. The result is the Gaussian distribution for $\vartheta_{0}$,

$$
\mathbb{P}\left(\vartheta_{0}\right) \propto \exp \left(-\frac{\vartheta_{0}^{2}}{2 \Sigma_{(2)}^{2}}\right)
$$

It remains to evaluate the variance $\Sigma_{(2)}^{2}$. In the present case, we have $\Sigma_{n}=\int_{0}^{\Lambda} \mathrm{d} k k^{2} \psi_{n}(k)$. From the completeness relation Eq. (A4), it follows that

$$
\sum_{n} k_{0}^{2} \psi_{n}\left(k_{0}\right) k^{2} \psi_{n}(k)=\frac{k^{2} \mathcal{P}\left(k_{0}\right) \mathcal{W}^{2}\left(k_{0}\right)}{k_{0}^{3}} \delta\left(k-k_{0}\right) .
$$

$\Sigma_{(2)}^{2}$ is now obtained by integrating term-by-term under the summation. The result coincides with the conventional smoothed variance,

$$
\Sigma_{\Lambda}^{2}\left(k_{H}\right)=\int_{0}^{\Lambda} \mathrm{d} \ln k \mathcal{W}^{2}\left(k ; k_{H}\right) \mathcal{P}(k)
$$

Thus, as expected, Eq. (B12) reproduces the Gaussian distribution (2) which was derived on the basis of the central limit theorem, with the proviso that the parameters (such as $\Sigma_{(2)}^{2}$ ) describing the distribution of $\vartheta_{0}$ are associated with the smoothed field $\mathcal{R}$.

For the case of the central second derivative we integrate this probability against the $\delta$-function containing the desired condition (13)

$$
\mathbb{P}\left(\vartheta_{2}\right)=\int[d \mathcal{R}] \mathbb{G}[\mathcal{R}] \delta\left[\mathcal{R}^{\prime \prime}(0)-\vartheta_{2}\right]
$$

Using again the expression of the $\delta$-function as an integral and the condition on the derivative in terms of the spherical harmonic coefficients we have:

$$
\mathbb{P}\left(\vartheta_{2}\right) \propto \int[d \mathcal{R}] \int d z \mathbb{G}[\mathcal{R}] \exp \left[\mathrm{i} z\left(\frac{3(2 \pi)^{3} \vartheta_{2}}{\sqrt{4 \pi}}+\sum_{n} \Sigma_{n}^{(4)}\left(\sqrt{\frac{4}{5}} a_{2 \mid n}^{0}+a_{0 \mid n}^{0}\right)\right)\right],
$$

where the factor $\Sigma_{n}^{(4)}$ is defined as

$$
\Sigma_{n}^{(4)}=\int d k k^{4} \psi_{n}(k)
$$

In the integral (B16) the terms with factors of $a_{0 \mid n}^{0}$ are

$$
\exp \left[-\frac{1}{4 \pi^{2}(2 \pi)^{3}} \sum_{n=1}^{\infty}\left|a_{0 \mid n}^{0}\right|+\mathrm{i} z \sum_{n=1}^{\infty}\left|a_{0 \mid n}^{0}\right| \Sigma_{n}^{(4)}\right] .
$$

Completing squares, this last expression is equal to

$$
\exp \left[-\frac{1}{4 \pi^{2}(2 \pi)^{3}} \sum_{n=1}^{\infty}\left(\left|a_{0 \mid n}^{0}\right|-\mathrm{i}(2 \pi)^{3} 2 \pi^{2} z \Sigma_{n}^{(4)}\right)^{2}-(2 \pi)^{3} \pi^{2} z^{2} \Sigma_{(4)}^{2}\right] .
$$


In the same way we can complete squares for the expansion factors $a_{2 \mid n}^{0}$ :

$$
\begin{aligned}
\exp & {\left[-\frac{1}{4 \pi^{2}(2 \pi)^{3}} \sum_{n=1}^{\infty}\left|a_{2 \mid n}^{0}\right|+\mathrm{i} z \frac{4}{5} \sum_{n=1}^{\infty}\left|a_{2 \mid n}^{0}\right| \Sigma_{n}^{(4)}\right] } \\
& =\exp \left[-\frac{1}{4 \pi^{2}(2 \pi)^{3}} \sum_{n=1}^{\infty}\left(\left|a_{0 \mid n}^{0}\right|-\mathrm{i}(2 \pi)^{3} \frac{4 \pi^{2}}{\sqrt{5}} z \Sigma_{n}^{(4)}\right)^{2}-(2 \pi)^{3} \pi^{2} \frac{4}{5} z^{2} \Sigma_{(4)}^{2}\right] .
\end{aligned}
$$

And finally one can also complete squares for the terms containing the variable $z$ which are independent of $a_{0 \mid n}^{0}$ and $a_{2 \mid n}^{0}$,

$$
\begin{aligned}
& \exp \left[-(2 \pi)^{3} \pi^{2}\left(\frac{9}{5}\right) z^{2} \Sigma_{(4)}^{2}+\mathrm{i} \frac{3(2 \pi)^{3}}{\sqrt{4 \pi}} \vartheta_{2} z\right]= \\
& \exp \left[-(2 \pi)^{3} \pi^{2}\left(\frac{9}{5}\right) \Sigma_{(4)}^{2}\left(z-\mathrm{i} \frac{5}{12 \sqrt{\pi^{5}}} \frac{\vartheta_{2}}{\Sigma_{(4)}^{2}}\right)^{2}-\frac{5}{2} \frac{\vartheta_{2}}{\Sigma_{(4)}^{2}}\right],
\end{aligned}
$$

where for simplification we have written

$$
\Sigma_{(4)}^{2}=\sum_{n=1}^{\infty}\left(\Sigma_{n}^{(4)}\right)^{2}
$$

To evaluate this variance of the second derivative we use again the property (B13) to integrate the complete summation and obtain

$$
\Sigma_{(4)}^{2}=\int_{0}^{\Lambda} d \ln k \mathcal{W}^{2}\left(k, k_{H}\right) \mathcal{P}(k) k^{4}
$$

So by making the change of variables

$$
\begin{aligned}
& a_{0 \mid n}^{0} \mapsto a_{0 \mid n}^{0}+\mathrm{i} 2 \pi^{2}(2 \pi)^{3} \Sigma_{n}^{(4)} z, \\
a_{2 \mid n}^{0} & \mapsto a_{2 \mid n}^{0}+\mathrm{i} \frac{4 \pi^{2}}{\sqrt{5}}(2 \pi)^{3} \Sigma_{n}^{(4)} z \\
\text { and } \quad z & \mapsto z+\mathrm{i} \frac{5}{12 \sqrt{\pi^{5}}} \frac{\vartheta_{2}}{\Sigma_{(4)}^{2}},
\end{aligned}
$$

we can perform all the integrals and eliminate the gaussian ones which contribute only up to an overall numerical factor subsequently absorbed by normalisation. The remaining factor expresses the probability of finding a perturbation $\mathcal{R}$ with a central second derivative of value $\vartheta_{2}$,

$$
\mathbb{P}\left[\mathcal{R}^{\prime \prime}(\mathbf{r}=0)=\vartheta_{2}\right] \propto \exp \left(-\frac{5 \vartheta_{2}^{2}}{2 \Sigma_{(4)}^{2}}\right)
$$

[1] Zel'Dovich, Y. B., \& Novikov, I. D. 1966, azh, 43, 758

[2] S. Hawking, "Gravitationally collapsed objects of very low mass," Mon. Not. Roy. Astron. Soc. 152 (1971) 75.

[3] B. J. Carr, "The Primordial Black Hole Mass Spectrum," Astrophys. J. 201 (1975) 1.

[4] W. H. Press and P. Schechter, "Formation of galaxies and clusters of galaxies by selfsimilar gravitational condensation," Astrophys. J. 187 (1974) 425.

[5] B. J. Carr, J. H. Gilbert and J. E. Lidsey, "Black hole relics and inflation: Limits on blue perturbation spectra," Phys. Rev. D 50 (1994) 4853 [arXiv:astro-ph/9405027].

[6] A. R. Liddle and A. M. Green, "Cosmological constraints from primordial black holes," Phys. Rept. 307 (1998) 125 [arXiv:grqc/9804034].

[7] Y. Sendouda, S. Nagataki and K. Sato, "Mass spectrum of primordial black holes from inflationary perturbation in the RandallSundrum braneworld: A limit on blue spectra," JCAP 0606 (2006) 003 [arXiv:astro-ph/0603509].

[8] I. Zaballa, A. M. Green, K. A. Malik and M. Sasaki, "Constraints on the primordial curvature perturbation from primordial black holes," JCAP 0703 (2007) 010 [arXiv:astro-ph/0612379]. 
[9] E. Bugaev and P. Klimai, "Constraints on power spectrum of density fluctuations from PBH evaporations," arXiv:astro$\mathrm{ph} / 0612659$.

[10] K. Kohri, D. H. Lyth and A. Melchiorri, "Black hole formation and slow-roll inflation," JCAP 0804 (2008) 038 [arXiv:0711.5006 [hep-ph]].

[11] Bicknell, G. V., \& Henriksen, R. N. 1979, Astrophys. J. , 232, 670

[12] I. Hawke and J. M. Stewart, "The Dynamics Of Primordial Black Hole Formation," Class. Quant. Grav. 19 (2002) 3687.

[13] I. Musco, J. C. Miller and L. Rezzolla, "Computations of primordial black hole formation," Class. Quant. Grav. 22 (2005) 1405 [arXiv:gr-qc/0412063].

[14] J. C. Niemeyer and K. Jedamzik, "Near-Critical Gravitational Collapse and the Initial Mass Function of Primordial Black Holes," Phys. Rev. Lett. 80 (1998) 5481 [arXiv:astro-ph/9709072]

[15] J. C. Niemeyer and K. Jedamzik, "Dynamics of Primordial Black Hole Formation," Phys. Rev. D 59 (1999) 124013 [arXiv:astro$\mathrm{ph} / 9901292]$.

[16] M. Shibata and M. Sasaki, "Black hole formation in the Friedmann universe: Formulation and computation in numerical relativity," Phys. Rev. D 60 (1999) 084002 [arXiv:gr-qc/9905064].

[17] Nadezhin, D. K., Novikov, I. D., \& Polnarev, A. G. 1978, azh, 55, 216

[18] A. G. Polnarev and I. Musco, "Curvature profiles as initial conditions for primordial black hole formation," Class. Quant. Grav. 24 (2007) 1405 [arXiv:gr-qc/0605122].

[19] A. Cooray and R. K. Sheth, "Halo models of large scale structure," Phys. Rept. 372 (2002) 1 [arXiv:astro-ph/0206508].

[20] A. V. Maccio', A. A. Dutton and F. C. v. Bosch, "Concentration, Spin and Shape of Dark Matter Haloes as a Function of the Cosmological Model: WMAP1, WMAP3 and WMAP5 results," arXiv:0805.1926 [astro-ph].

[21] Polnarev, A. G., and Khlopov, M. Y. 1981, Soviet Astronomy, 25, 406

[22] Polnarev, A. G., and Khlopov, M. Y. 1982, Soviet Astronomy, 26, 391

[23] Khlopov, M. Y., \& Polnarev, A. G. 1983, Very Early Universe, 407

[24] Polnarev, A. G., \& Khlopov, M. Y. 1985, Uspekhi Fizicheskikh Nauk, 145, 369

[25] Zabotin, N. A., Naselskii, P. D., and Polnarev, A. G. 1987, Soviet Astronomy, 31, 353

[26] A. M. Green, A. R. Liddle, K. A. Malik and M. Sasaki, "A new calculation of the mass fraction of primordial black holes," Phys. Rev. D 70 (2004) 041502 [arXiv:astro-ph/0403181].

[27] A. R. Liddle and D. H. Lyth, "Cosmological inflation and large-scale structure," Cambridge Univ. Pr. (2000) $400 \mathrm{p}$

[28] D. S. Salopek and J. R. Bond, "Nonlinear evolution of long wavelength metric fluctuations in inflationary models," Phys. Rev. D 42 (1990) 3936

[29] D. H. Lyth, K. A. Malik and M. Sasaki, "A general proof of the conservation of the curvature perturbation," JCAP 0505 (2005) 004 [arXiv:astro-ph/0411220].

[30] D. Wands, K. A. Malik, D. H. Lyth and A. R. Liddle, "A new approach to the evolution of cosmological perturbations on large scales," Phys. Rev. D 62 (2000) 043527 [arXiv:astro-ph/0003278].

[31] N. Deruelle and D. Langlois, "Long Wavelength Iteration Of Einstein's Equations Near A Space-Time Singularity," Phys. Rev. D 52 (1995) 2007 [arXiv:gr-qc/9411040].

[32] D. Langlois and F. Vernizzi, "Evolution of non-linear cosmological perturbations," Phys. Rev. Lett. 95 (2005) 091303 [arXiv:astro$\mathrm{ph} / 0503416]$.

[33] G. I. Rigopoulos and E. P. S. Shellard, "Non-linear inflationary perturbations," JCAP 0510 (2005) 006 [arXiv:astro-ph/0405185].

[34] D. Langlois and F. Vernizzi, "Conserved non-linear quantities in cosmology," Phys. Rev. D 72 (2005) 103501 [arXiv:astro$\mathrm{ph} / 0509078]$.

[35] E. M. Lifshitz and I. M. Khalatnikov, "Investigations in relativistic cosmology," Adv. Phys. 12 (1963) 185.

[36] David Seery and Carlos Hidalgo, "Non-Gaussian corrections to the probability distribution of the curvature perturbation from inflation," JCAP 0607, 008 (2006) [arXiv:astro-ph/0604579].

[37] M. LoVerde, A. Miller, S. Shandera and L. Verde, "Effects of Scale-Dependent Non-Gaussianity on Cosmological Structures," JCAP 0804 (2008) 014 [arXiv:0711.4126 [astro-ph]].

[38] X. Kang, P. Norberg and J. Silk, "Can large-scale structure probe CMB-constrained non-Gaussianity?," Mon. Not. Roy. Astron. Soc. 376 (2007) 343 [arXiv:astro-ph/0701131].

[39] S. Matarrese, L. Verde and R. Jimenez, "The abundance of high-redshift objects as a probe of non-Gaussian initial conditions," Astrophys. J. 541 (2000) 10 [arXiv:astro-ph/0001366].

[40] J. S. Bullock and J. R. Primack, "Non-Gaussian fluctuations and primordial black holes from inflation," Phys. Rev. D 55 (1997) 7423 [arXiv:astro-ph/9611106].

[41] P. Ivanov, "Non-linear metric perturbations and production of primordial black holes," Phys. Rev. D 57 (1998) 7145 [arXiv:astro$\mathrm{ph} / 9708224]$.

[42] J. C. Hidalgo, "The effect of non-Gaussian curvature perturbations on the formation of primordial black holes," arXiv:0708.3875 [astro-ph].

[43] R. Saito, J. Yokoyama and R. Nagata, "Single-field inflation, anomalous enhancement of superhorizon fluctuations, and nonGaussianity in primordial black hole formation," arXiv:0804.3470 [astro-ph].

[44] X. Chen, M. x. Huang, S. Kachru and G. Shiu, "Observational signatures and non-Gaussianities of general single field inflation," JCAP 0701 (2007) 002 [arXiv:hep-th/0605045].

[45] X. Chen, R. Easther and E. A. Lim, "Large non-Gaussianities in single field inflation," JCAP 0706 (2007) 023 [arXiv:astro$\mathrm{ph} / 0611645]$.

[46] D. H. Lyth and Y. Rodriguez, "The inflationary prediction for primordial non-gaussianity," Phys. Rev. Lett. 95 (2005) 121302 [arXiv:astro-ph/0504045].

[47] D. Seery and J. E. Lidsey, "Primordial non-gaussianities in single field inflation," JCAP 0506 (2005) 003 [arXiv:astro-ph/0503692].

[48] L. I. Landau, E. m. Lifshitz, "Classical Field Theory," Oxford, Uk: Pergamon ( 1994) 402 P. (Course Of Theoretical Physics, 2)

[49] Y. B. Zeldovich and I. D. Novikov, "Relativistic Astrophysics. Vol. 2. The Structure And Evolution Of The Universe," Chicago, Usa: Chicago Univ. (1983) $718 p$

[50] H. Kodama and M. Sasaki, "Cosmological Perturbation Theory," Prog. Theor. Phys. Suppl. 78 (1984) 1.

[51] Y. Tanaka and M. Sasaki, "Gradient expansion approach to nonlinear superhorizon perturbations," Prog. Theor. Phys. 117 (2007) 633 [arXiv:gr-qc/0612191].

[52] M. Shibata and H. Asada, "PostNewtonian equations of motion in the flat universe," Prog. Theor. Phys. 94, 11 (1995).

[53] B. J. Carr, "Primordial black holes - recent developments," In the Proceedings of 22nd Texas Symposium on Relativistic Astrophysics at Stanford University, Stanford, California, 13-17 Dec 2004, pp 0204 [arXiv:astro-ph/0504034].

[54] S. Chongchitnan and G. Efstathiou, "Accuracy of slow-roll formulae for inflationary perturbations: Implications for primordial black hole formation," JCAP 0701 (2007) 011 [arXiv:astro-ph/0611818].

[55] E. Bugaev and P. Klimai, "Large curvature perturbations near horizon crossing in single-field inflation models," arXiv:0806.4541 [astro-ph]. 